\title{
A Model for Assessing the Quantitative Effects of Heterogeneous Affinity in Malaria Transmission along with Ivermectin Mass Administration
}

\author{
João Sequeira ${ }^{1,2} \mathbb{D}$, Jorge Louçã ${ }^{1}$, António M. Mendes ${ }^{3}\left(\mathbb{D}\right.$ and Pedro G. Lind ${ }^{4,5, *(D)}$ \\ 1 ISTAR-IUL, Instituto Universitário de Lisboa (ISCTE-IUL), Av. das Forças Armadas, \\ 1649-026 Lisboa, Portugal; jsequeira@chlo.min-saude.pt (J.S.); jorge.1@iscte-iul.pt (J.L.) \\ 2 Hospital Santa Cruz, Av. Reinaldo dos Santos, 2790-134 Carnaxide, Portugal \\ 3 Instituto de Medicina Molecular, Faculdade de Medicina, Universidade de Lisboa, Av. Egas Moniz, \\ 1649-028 Lisboa, Portugal; antoniomendes@medicina.ulisboa.pt \\ 4 Department of Computer Science, OsloMet-Oslo Metropolitan University, P.O. Box 4 St. Olavs Plass, \\ N-0130 Oslo, Norway \\ 5 OsloMet AI Lab, Pilestredet 52, N-0166 Oslo, Norway \\ * Correspondence: pedro.lind@oslomet.no
}

Received: 12 October 2020; Accepted: 30 November 2020; Published: 4 December 2020

\begin{abstract}
Using an agent-based model of malaria, we present numerical evidence that in communities of individuals having an affinity varying within a broad range of values, disease transmission may increase up to $300 \%$. Moreover, our findings provide new insight into how to combine different strategies for the prevention of malaria transmission. In particular, we uncover a relationship between the level of heterogeneity and the level of conventional and unconventional anti-malarial drug administration (ivermectin and gametocidal agents), which, when taken together, will define a control parameter, tuning between disease persistence and elimination. Finally, we also provide evidence that the entomological inoculation rate, as well as the product between parasite and sporozoite rates are both good indicators of malaria incidence in the presence of heterogeneity in disease transmission and may configure a possible improvement in that setting, upon classical standard measures such as the basic reproductive number.
\end{abstract}

Keywords: malaria transmission; $R_{0}$; EIR; heterogeneity; hotspots; ivermectin

\section{Introduction}

Since the turn of the century, world malaria annual incidence decreased from 14.6 cases per 100 human inhabitants (pHHI), in 2000 [1], to 5.7 cases, in 2018 [2]. This improvement is the result of adequate public health policy involving preventive actions such as vector control, implementation of rapid diagnostic testing (RDT), drug administration of artemisinin combined therapy (ACT), widespread use of barrier protection measures such as long-lasting insecticide-treated bed nets (LLIN), and mixed barrier protection through vector control measures such as indoor insecticide residual spraying (IRS). However, the risk of malaria resurgence still represents a constant menace in regions where disease transmission was assumed to be previously controlled. During the year 2018, sub-Saharan African malaria incidence was still unacceptably high, at a rate of 22.9 annual cases pHHI [2]. The causes for these resurgence scenarios are still unclear and appeal to more realistic models that may uncover the essential mechanisms leading to them.

The presence of heterogeneity in malaria may influence disease transmission in multiple ways [3]. It may concern different human and vector densities, several forms of diversity in human age distribution, cultural, social, and genetic population backgrounds, as well as a variable 
host-acquired immunity from previous infections. It may also relate to differences in climate conditions, land topography, local altitudes, housing structure, human migration, and the unequal use of preventive and treatment measures [4,5]. Indeed, heterogeneous mosquito biting has been reported as a possible factor for enhancing malaria transmission: human hosts who are bitten most will later on infect a larger number of mosquitoes [6] and will be more likely to behave simultaneously as super-receivers, as well as super-spreaders [7,8]. Moreover, not all human beings are equally attractive to mosquitoes. Multiple factors seem to influence the mosquito feeding routine. During day time, the mosquito vision apparatus seems to be the most effective mosquito guidance system. However, at night time, olfactory cues may play a more important role in mosquito host-seeking behavior. The affinity of the disease vector Anopheles is related to the presence of specific odors in the human host, namely different chemical stimuli called kairomones such as the $\mathrm{CO}_{2}$ percentual fraction in human exhaled air or the presence of some aliphatic carboxylic acids in the human skin. Human $\mathrm{CO}_{2}$ breathing may be the single most important factor conditioning mosquito feeding preferences [5]. Furthermore, different Anopheles subspecies may display different levels of anthropophillic mosquito feeding habits [9-12]. Furthermore, infectious hosts may display higher mosquito bite attractiveness as a result of the presence of high levels of gametocytemia [13-15].

However, till the 90's, most of the models of malaria dynamics assumed homogeneous transmission, while a precise and objective quantitative approach to realistic scenarios was still lacking. In 1997, Woolhouse defined a specific model for the presence of heterogeneity in malaria transmission, naming it the $20 / 80$ rule [16]. This rule assumes that $80 \%$ of all mosquito bites will occur in $20 \%$ of the human population, being inspired by Wilfredo Pareto's original work on the distribution of world wealth [17]. This smaller super-spreader subgroup [8] concentrates the effect of repeated biting leading to a proportional increase in the risk of disease transmission to human hosts. Furthermore, it stands as a privileged reservoir with a higher probability of mosquito reinfection as a result of higher gametocytemia levels. In identical endemic conditions, this fact alone may increase several-fold the probability of disease transmission [16]. We will refer to this Pareto-Woolhouse rule as the PW-rule. For a review comparing the major malaria transmission models, see [18].

Spatial variation in malaria incidence and exposure to infected mosquitoes in Tanzania has been related to the presence of clusters of higher malaria incidence among infants [19]. These were defined as hotspots of malaria transmission, with higher mosquito bite exposure. In this case, while a small group of children (around 10\%) experienced several malaria episodes, there was no clinical evidence of malaria symptoms in two thirds of all children. A stronger human exposure to Anopheles mosquito may also result from spatial factors such as a closer distance to vector breeding sites, prolonged outdoor human activities, and human genetics responsible for the production of odors more attractive to mosquitoes [20]. In some African villages, the existence of hotspots supports continuing transmission during the dry season, while acting as a source of infection for the rest of the village during the wet season [20]. Within a few districts of Bangladesh with stable hotspots, the malaria incidence distribution was remarkably similar to the heterogeneity of the $20 / 80$ rule described by Woolhouse [16,21].

In Figure 1, it is possible to see that human and mosquito infection prevalence time series are clearly influenced by model heterogeneity. Comparing two typical scenarios, one in which the number of bites is distributed uniformly among the human population (Figure 1a) and another where the PW-rule of 20/80 is applied (Figure $1 b$ ), the model predicts that heterogeneity clearly promotes malaria persistence in both mosquito and human populations.

In this paper, we provide quantitative evidence that malaria spreading effectiveness is strongly sensitive to heterogeneous affinity. Section 2 describes the agent-based malaria model used in simulating different scenarios of malaria spreading, introducing a parameter for measuring heterogeneous affinity. In Section 3.1, we investigate the possible transitions from elimination to disease persistence, focusing on the role of three parameters: heterogeneous affinity, level of drug administration, i.e., the fraction of human population at risk undertaking ivermectin treatment, and human-to-mosquito (H-to-M) transmission efficiency. In particular, we use a model validation 
procedure evaluating the impact of human ivermectin treatment in mosquito survival, when compared to similar results obtained from previous research [22,23]. In Section 3.2, we discuss further which measurable quantities can in real situations better predict malaria incidence. Section 4 concludes the paper.
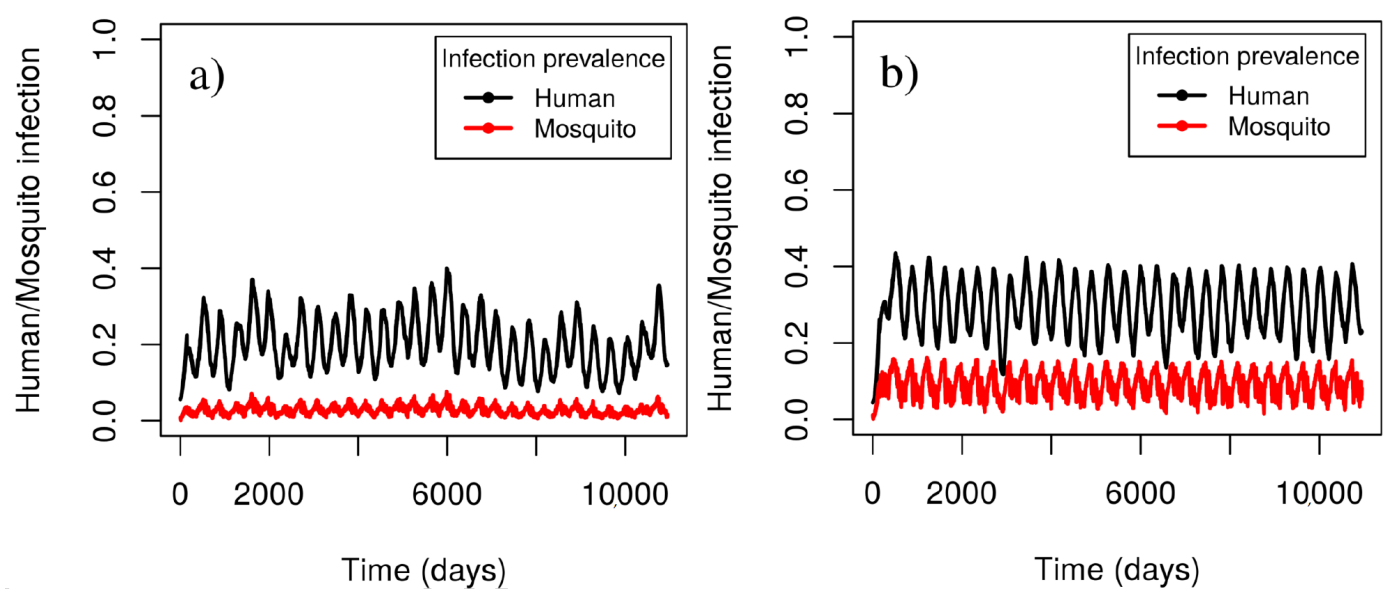

Figure 1. Human and mosquito infection prevalence (a) in a scenario of homogeneous affinity, namely $20 \%$ of the human individuals receive $20 \%$ of mosquitoes bites, and (b) in a heterogeneous scenario when $20 \%$ of the human individuals receive $80 \%$ of mosquitoes bites. In both cases, intermediate human-to-mosquito transmission efficiency is considered, $w_{h}=0.500$.

\section{Methods, Model, and Analysis Tools}

There are many factors influencing heterogeneous affinity in mosquito and human populations. Some of the most important are the existence of hotspots, genetic propensity, migratory human behavior, and particular geophysical conditions. Instead of considering these different factors separately, we will look at their collective effect in a small fraction of human individuals receiving the majority of mosquito bites. In other words, we will parameterize a sort of Pareto rule in its more modern formulation, according to Woolhouse [16].

\subsection{Implementing Heterogeneity in Malaria Spreading}

Our model extends a previous agent-based malaria model introduced in [24], now incorporating heterogeneous affinity. We consider a system of $N_{m}=4000$ mosquitoes and $N_{h}=2000$ human individuals, both including healthy and infected individuals, henceforth represented as $M_{0}$ and $H_{0}$ and as $M_{i}$ and $H_{i}$, respectively $\left(N_{m}=M_{0}+M_{i}\right.$ and $\left.N_{h}=H_{0}+H_{i}\right)$. Although the density of mosquitoes in the field may be higher than the one considered here, we model the number of mosquitoes as a smaller effective fraction of the overall mosquito mass that randomly bites a human individual, on average, twice a day, with a uniform distribution, and ranging from no bites to four daily bites. The algorithm keeps track of all attributes for each agent, human or mosquito, for a particular age, time since infection, and immunity status. All simulations take place during a time period of 30 years. More details and a flowchart describing the computer implementation of the agent-based model are given in [24].

Heterogeneous affinity is here defined by the PW-rule parameter:

$$
\theta=\frac{P_{h}}{P_{b}}
$$

giving the quotient between the percentage $P_{h}$ of the group with the most bitten humans and the percentage $P_{b}$ of the bites that the group will receive. Next, we describe the relation between the basic reproduction number of the Ross-Macdonald model $[25,26]$ and the heterogeneity affinity rule 
introduced above. The basic reproductive number $R_{0}$ represents the number of secondarily infected humans that result from a single infectious human, and it is defined as:

$$
R_{0}=\frac{m a^{2} b c}{q_{h} q_{m}}(1+\alpha)=\bar{R}_{0}(1+\alpha)
$$

where $m$ is the mosquito density, $a$ is the human feeding rate, $b$ and $c$ represent respectively the mosquito-to-human (M-to-H) and human-to-mosquito (H-to-M) transmission efficiencies, and $q_{h}$ and $q_{m}$ are, respectively, the human daily recovery rate and the mosquito daily mortality rate. Parameter $\alpha \geq 0$ was used in previous studies accounting for the effects of heterogeneous affinity $[3,5,8,16,20,27-29]$ and corresponds to the so-called index of biting disparity, which has been included by assuming that the previous basic reproductive number corresponds to the homogeneous basic reproductive number $\bar{R}_{0}$.

From empirical studies $[8,16,19,20]$, parameter $\alpha$ is known to assume values in the range $0<\alpha<$ $\alpha_{\max } \lesssim 4$. Next, we introduce an explicit definition for $\alpha$, to map it from parameter $\theta$ :

$$
\alpha=\alpha_{\max }\left(1-\exp \left(-\frac{(\log \theta)^{2}}{2 \sigma^{2}}\right)\right)
$$

where $\sigma=|\log (20 / 80)|$ to settle the PW-rule of $20 / 80$ as the heterogeneous affinity deviating from the homogeneous state by one standard deviation, and $\alpha_{\max }=4$. A sketch of $\alpha$ as defined in Equation (3) as a function of $\theta$, while Equation (1), is shown in the inset of Figure 2.

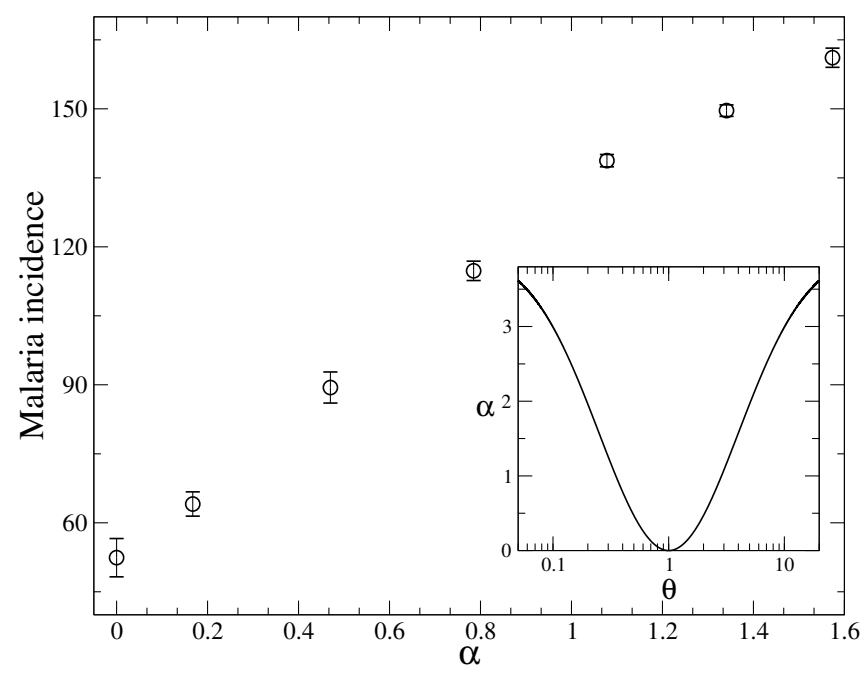

Figure 2. Malaria incidence as a function of heterogeneous affinity $\alpha$ as defined in Equation (3). The inset shows the change in heterogeneous affinity with $\theta$, as defined in Equation (1).

In the present paper, the authors intend to define a specific metric for heterogeneity quantification in the form of its possible impact on local disease transmission, equivalent to the effect of a small migrating gametocyte-carrier population in the increase of disease resurgence risk. The utility of preventive treatment with ivermectin in the context of high heterogeneity in disease transmission while in the presence of different levels of background gametocytemia in the human population is also analyzed in terms of effective disease prevention. This intervention may be used as an adjuvant therapy to other anti-human-to-mosquito transmission measures resulting in gametocytemia reduction, by the use of effective drugs such as artemisinin, primaquine, or methylene blue [30-33]. Therefore, following also our previous study in [24], in this paper, we also consider two important aspects of malaria spreading in the presence of heterogeneity, which enable us to use our previous results in the discussion of a possible new insight into improving the control of malaria transmission. The two aspects are (i) gametocytemia reduction by the use of specific treatment with primaquine, methylene blue, or artemisinin derivatives and (ii) ivermectin prevention. 


\subsection{Human-to-Mosquito Transmission and Drug Administration in a Heterogeneity Setting}

At least two mosquito bites are required to complete the human-mosquito-human malaria transmission cycle. However, it is the presence of gametocytes in the blood at the human-to-mosquito transmission stage that stands as the most critical step in the persistence of malaria transmission.

Because human hosts can present a wide range of gametocytemia levels on different occasions and during a finite period of time, the effectiveness of human-to-mosquito transmission assumes a stochastic nature, conditioning some form of heterogeneity in the process. In the present model, human-to-mosquito transmission efficiency $w_{h}$ is defined as the fraction between the number $\tau_{g}$ of days with positive gametocytemia and the expected total duration $\tau_{d}$ of the disease, as [24]:

$$
w_{h}=\frac{\tau_{g}}{\tau_{d}}
$$

Here, we consider the typical value $\tau_{d}=150$ days. The time period with potential gametocyte production is usually longer than the disease symptomatic phase, which is of a shorter duration. The assumed value of 150 days represents a reasonable approximation to the upper range of the expected average disease duration. Gametocytemia duration was defined as an essential model parameter, tunable through a dynamically equivalent action by the potential use of a gametocidal drug agent. In the present model, heterogeneity is analyzed at different levels of disease $\mathrm{H}$-to-M transmission (from $w_{h}=0.400$ to $w_{h}=0.800$ ).

In the case of heterogeneous transmission, the combination of several anti-malarial strategies is crucial to achieve epidemic control [3]. The possibility of quarantine may be ineffective in malaria asymptomatic patients. Even gametocyte carriers may briefly show only asexual forms of the most dangerous parasite Plasmodium falciparum $(P f)$ in their peripheral blood. With new laboratory assays for early disease diagnosis, diagnostic accuracy in migrant populations may improve. However, deciding which migrants should be treated with effective anti-malaria treatment at admittance is debatable.

Ivermectin has proven effective against Plasmodium falciparum infection, by selective killing of Anopheles mosquitoes shortly after a successful bite, before the parasite is able to complete its sporogonic development [22,34-39], and apparently without any form of direct toxicity to the parasite [40]. This ivermectin selective killing action on biting mosquitoes may be extremely effective in disease prevention, by reducing the need for treatment with artemisinin derivatives, thus lowering the risk of artemisinin drug-resistance expansion [37].

Ivermectin prevention was introduced in the present model as an adjustable parameter $\delta_{i v}$, according to a previously published definition [24]. The $\delta_{i v}$ parameter results from the product of ivermectin administration probability $p_{i v}$ (in the range between 0.0 , with no ivermectin prevention, and 0.50 , with ivermectin prevention in $50 \%$ of the human population) and the ivermectin-induced mosquito mortality constant parameter $\left(g_{i v}=0.50\right)$ :

$$
\delta_{i v}=p_{i v} \times g_{i v} .
$$

This mathematical implementation of the ivermectin effect is based on the knowledge that approximately half of all mosquitoes will die during the first four days after feeding on a human-host under ivermectin treatment [22]. As described below, we will validate such implementations in our model, looking into mosquito survival distribution curves and comparing them with Chaccour's original results. We also evaluate if there is any correlation between mosquito mortality as a result of ivermectin human treatment when compared to mosquito mortality occurring during the parasite incubation period inside the mosquito, according to what would be expected from theoretical predictions.

At this point, we clarify a few aspects to address the limitations of our approach and better establish the context of this agent-based simulation scenario. In order to include the effect of ivermectin 
in our model, we needed to use a simplified computational procedure replicating the consequences of mosquito feeding on human individuals under ivermectin treatment. Although this procedure may be considered overly simplistic, it is, none the less, computationally efficient, being based on a simple Bernoulli process in order to decide the fate of the mosquito after feeding on a human individual under ivermectin. In this way, one does not take into account aspects that could be addressed in the clinical context, namely ivermectin pharmacokinetics and pharmacodynamics [23]. Our validation procedure focuses on comparing mosquito survival data from our model simulation with data from empirical mosquito survival data, such as presented in [22]. We did not include any other pharmacokinetic concept in our computational algorithm, as we considered the assumption from published data, that $50 \%$ of mosquitoes would die within four days after feeding on a human host under ivermectin treatment, valid. In our computational algorithm, we pretend to simulate ivermectin toxicity with a simpler procedure, ignoring other more complex pharmacokinetic concepts. The impact of ivermectin in inducing mild mosquito mortality in the time spent during disease incubation is, most likely, the critical factor in reducing disease transmission with ivermectin treatment, as the effect of ivermectin in global mosquito density is mainly residual. Our validation procedure was essentially based on determining the mosquito survival distribution curves from our model and whether they would be compatible with the expected results obtained from our pharmacokinetic assumptions for the mosquito survival distribution curves, when compared to empirical data, such as presented in [22].

\subsection{Predictive Rates for Assessing the Strength of Malaria Transmission and the Annual Entomological Inoculation}

Field malaria transmission is difficult to monitor, requiring various methods to measure different aspects of disease transmission [41]. Here, we consider three metrics to monitor the intensity of malaria transmission: (a) malaria incidence $(M I)$, (b) parasite rate $(X)$ for human individuals, and (c) sporozoite rate $(Z)$ for mosquitoes.

Malaria incidence is defined as the confirmed malaria cases per person per unit of time and, in particular, as the annual number of confirmed malaria cases per 100 inhabitants at risk. The parasite rate $X$ is given by the fraction of infected humans and is also known as the prevalence of infection in humans [6,42]. This rate is obtainable from the slide positivity rate (SPR), which is defined as the number of microscopy-confirmed malaria tests per 100 suspected cases examined [43]. SPR is useful in establishing temporal trends in malaria and simple to implement at peripheral health facilities. It only considers laboratory confirmed cases of malaria and may also be derived from rapid diagnostic testing (RDT-PR) [44]. SPR has been associated with malaria incidence and identified as a strong predictor of malaria transmission in Yunnan province, China [45]. The sporozoite rate $Z$ represents the fraction of infected mosquitoes $[42,46]$.

Although these two indices, $Z$ and $X$, are commonly used separately in malaria research [42], we combined their behavior with respect to heterogeneity, introducing a "global infection rate" $G$ parameter, which is given by the product of both rates:

$$
G \sim Z X
$$

where the pre-factor is introduced in order to have the result in the form of a squared percentage.

Related to these predictive rates is the annual entomological inoculation rate (EIR) defined as:

$$
\mathrm{EIR}=365 \mathrm{maZ}
$$

where, as stated above, $m$ is the mosquito density and $a$ is the human feeding rate. 


\section{Results}

\subsection{From Disease Persistence to Elimination in a Scenario of Increasing Heterogeneity}

\subsubsection{Heterogeneity and Ivermectin}

The present model was implemented for sets of ten simulations in three heterogeneity scenarios, defined by the following values of heterogeneity affinity: low $(\theta=1,0.66)$, intermediate $(\theta=0.5,0.4)$, and strong $(\theta=0.33,0.29,0.25)$. See Equation (1). These values are equivalent to the set of PW-rules of $20 / 20,20 / 30,20 / 40,20 / 50,20 / 60,20 / 70$, and 20/80, respectively. Our results show a consistent linear correlation between 30 year average malaria incidence and increasing levels of heterogeneity, suggesting that heterogeneity strongly promotes malaria transmission (Figure 2).

Next, we analyze how heterogeneity in disease transmission is influenced by ivermectin treatment, i.e., by the level of drug administration. In the absence of ivermectin administration, malaria incidence is three times higher in high heterogeneity simulations related to the PW-rule of 20/80 $(\theta=0.25)$, for which around 150 annual cases pHHI are observed, when compared to simulations in full homogeneity $(\theta=1.0)$ where one observes $\sim 50$ annual cases $\mathrm{pHHI}$. The curve in Figure 3a for $p_{i v}=0$ clearly shows this pattern. Moreover, this pattern is significantly reduced if ivermectin is used by even a small fraction of $5 \%$ of the general population, and a major decline in malaria incidence, very close to disease extinction, can be achieved by using ivermectin treatment in $20 \%$ of the general population, in the high heterogeneity scenario (PW-rule of $20 / 80, \theta=0.25$ ).

An important feature is revealed in Figure $3 b$ : isolines of constant malaria incidence fit approximately a linear relation $\alpha+s p_{i v}$ with $s \simeq-18$. If malaria incidence is plotted as a function of this linear relation, we uncover a transition from disease persistence to elimination at $\alpha-18 p_{i v} \sim-1$. Our results suggests that an increase in malaria transmission due to heterogeneity can be overcome when the population fraction under ivermectin treatment is such that $p_{i v} \gtrsim(\alpha+1) / 18$.

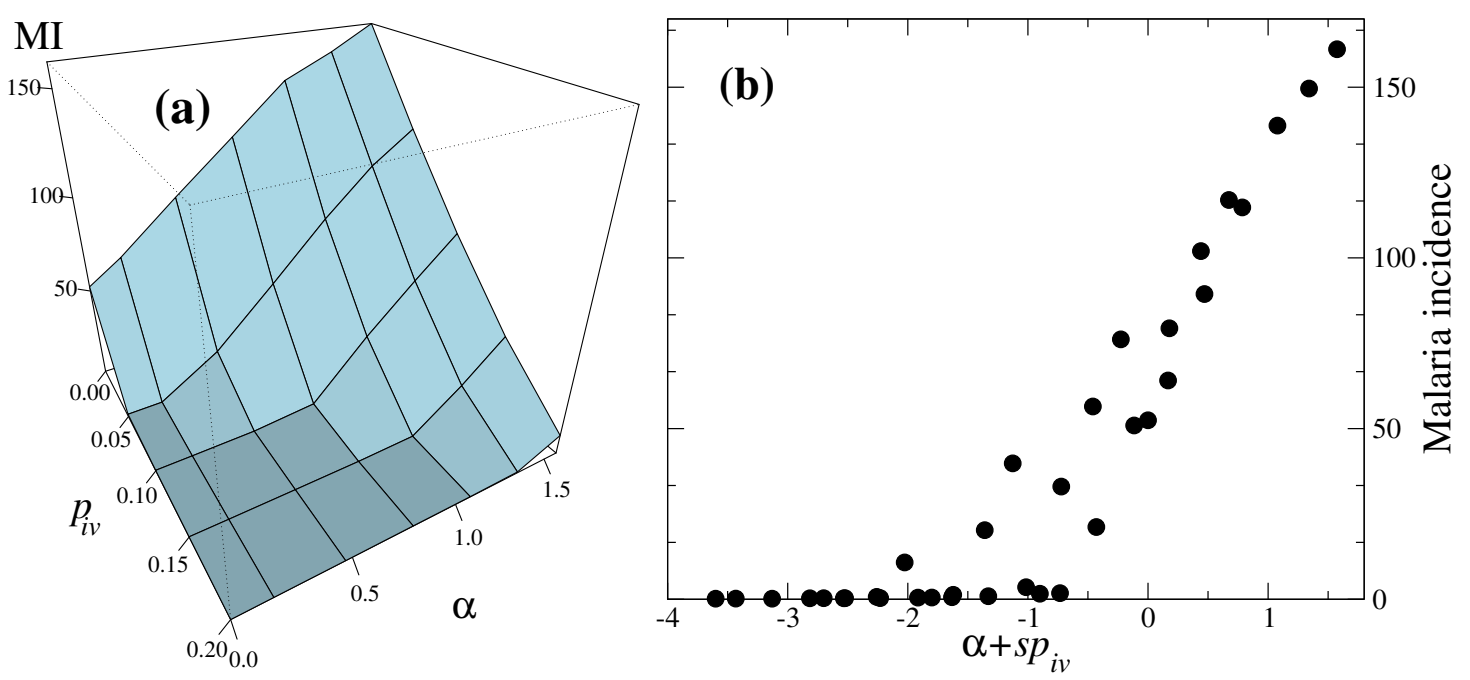

Figure 3. (a) Malaria incidence as a function of the heterogeneous affinity $\alpha$ and the ivermectin probability $p_{i v}$, showing that isolines of malaria spreading are approximately a linear combination of both variables. (b) Malaria incidence as a function of the linear combination $\alpha+s P_{i v}$ with $s=-18$, showing a transition from disease persistence to elimination. In all cases, the human-to-mosquito transmission efficiency is held constant at $w_{h}=0.5$.

It is important to stress that our model predicts a higher impact of ivermectin in disease transmission when compared to previous empirical results [38]. Our agent-based model is a simplified version of real events concerning the consequences of iterating mosquito bites in human individuals, in a closed and well controlled simulation environment. However, despite this limitation as a tool for exploring hypothetical scenarios, the ivermectin algorithm in the model simulation presents 
realistic results at a more qualitative level, in compliance with the expected ivermectin-induced mosquito mortality.

3.1.2. The Role of Transmission Efficiency Combined with Ivermectin Treatment in the Heterogeneous Scenario

In the present model, human-to-mosquito transmission efficiency $w_{h}$ is related to the time of gametocytemia duration and reveals a strong correlation with malaria incidence in high heterogeneity scenarios. However, this correlation is consistently suppressed with higher rates of ivermectin prevention, as shown in Figure 4a.

In this case, as shown in Figure 4b, isolines of constant malaria incidence fit approximately a linear relation $w_{h}+s p_{i v}$ with $s \simeq-4.2$, and malaria incidence is approximately proportional to this linear combination.
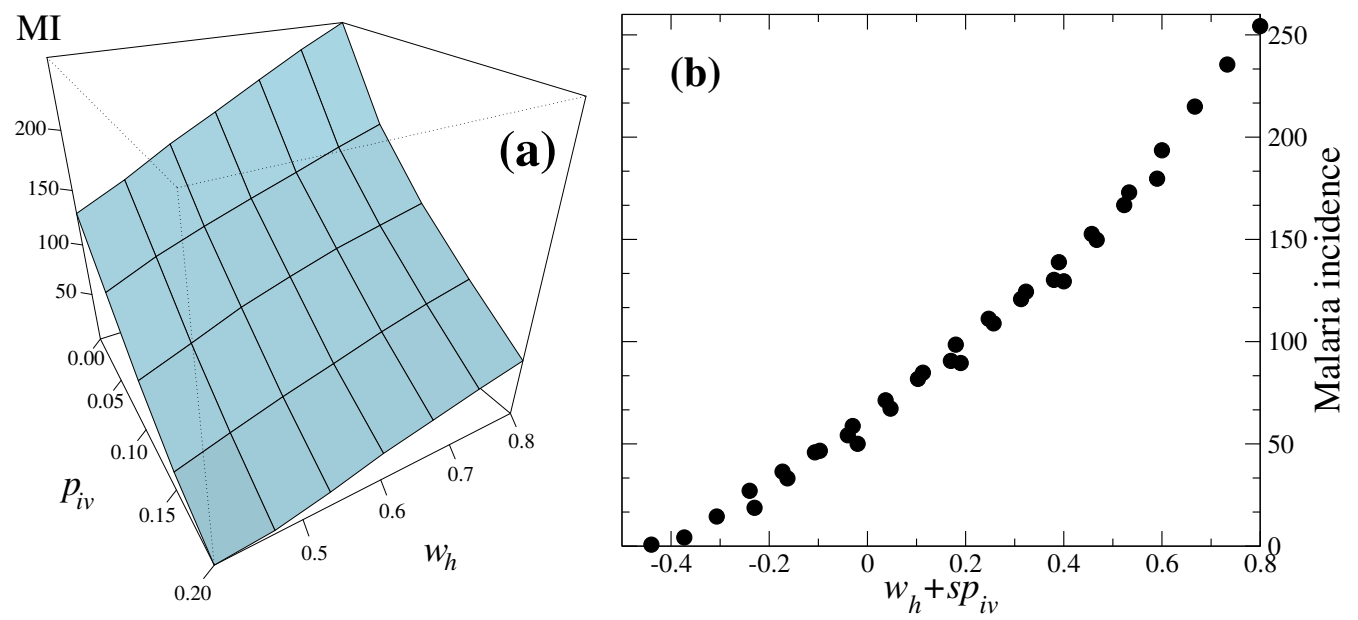

Figure 4. (a) Malaria incidence as a function of the human-to-mosquito transmission efficiency $w_{h}$ and the ivermectin probability $p_{i v}$, showing that isolines of malaria spreading are approximately a linear combination of both variables. Therefore, we plot in (b) the malaria incidence as a function of the linear combination $w_{h}+s P_{i v}$ with $s=-4.2$, clearly showing a gradual transition from elimination to endemic prevalence. In all cases, the PW-rule of 20/80 was used, corresponding to $\theta=0.25$.

\subsubsection{Mosquito Survival Patterns}

In the present model, ivermectin is responsible for a consistent reduction in mosquito life expectancy resulting in lower chances for Plasmodium parasites to mature to the stage of sporozoite inside the mosquito, after feeding on a gametocyte-carrier human host. With a higher fraction of human hosts on ivermectin treatment, mosquito mortality is higher during the initial period of the mosquito life time before the necessary ten days for parasite maturation inside the mosquito gut. See Figure 5.

Average mosquito mortality, during the 10 day parasite incubation time period as a direct result of ivermectin treatment, is influenced by the fraction of human hosts on ivermectin treatment. Mosquito mortality was consistently stable and independent of different heterogeneity levels in all simulations with identical model parameters. In Figure 5a, we show the mosquito survival distribution curve for ta 30 year time for different values of ivermectin probability and fit it with an exponential function:

$$
N=A \exp (\gamma t)
$$

with $\gamma$ and $A$, which vary linearly with $p_{i v}$, as shown in Figure $5 b$,c.

The intersection point in Figure 5a around 10 days coincides approximately with the parasite incubation time period inside the mosquito, as ivermectin-induced mosquito mortality is typically 
higher during the first ten days of treatment and becomes residual thereafter. The incubation time period found in our simulation is of the same order of magnitude of the value found in other studies [37].
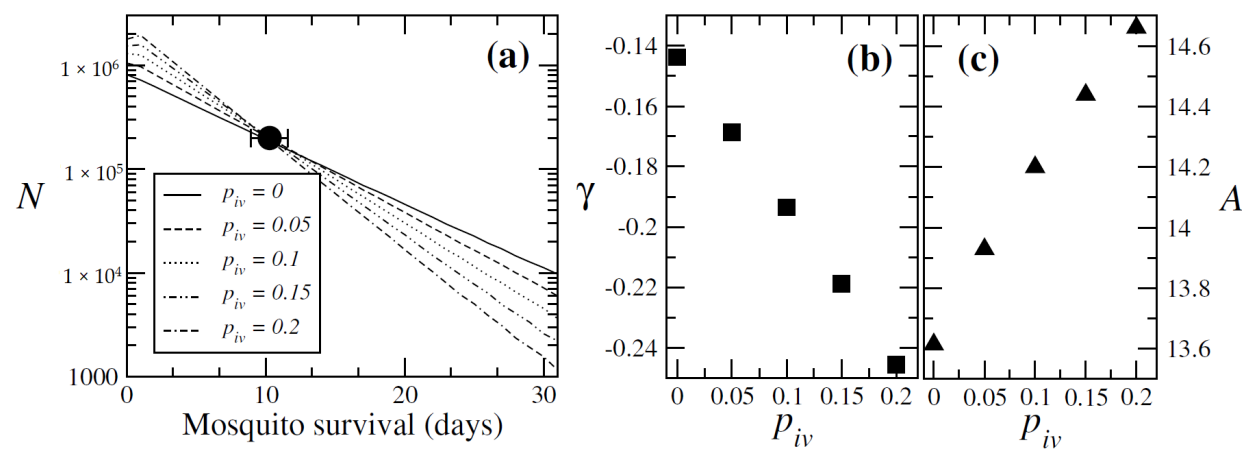

Figure 5. (a) Mosquito survival distribution curve for 30 year time series (five samples), uncovering an intersecting point around 10 days. The exponential fit $N=A \exp (\gamma t)$ has parameters, $\gamma$ and $A$, which vary linearly with $P_{i v}$ as shown in $(\mathbf{b}, \mathbf{c})$. In all cases, high heterogeneity $(\theta=0.25)$ and low human-to-mosquito transmission efficiency $\left(w_{h}=0.5\right)$ are fixed.

\subsubsection{Validation of the Ivermectin Parameter}

A theoretical approach to model validation is usually difficult to implement when evaluating a computational algorithm equivalent to the use of ivermectin in a human population. Still, in this section, we describe a model validation procedure by graphically comparing the results from our model, incorporating ivermectin as a quantitative parameter in terms of mosquito survival, with data from previous research [22].

As shown in Figure 6, the differences found in mosquito survival between the ivermectin human treatment group and the human control group are small. However, notice that mosquito survival at 12 days is slightly higher in the model simulation, close to $20 \%$, when compared to the residual survival in the empirical data [22]. This difference can be explained by the implementation of a more conservative computational algorithm in our model, in terms of mosquito survival. Globally, our model survival curves were quite similar in shape to those of Chaccour's original paper, suggesting that this ivermectin model may reproduce acceptable model simulations, with mosquito survival results within the expected range.

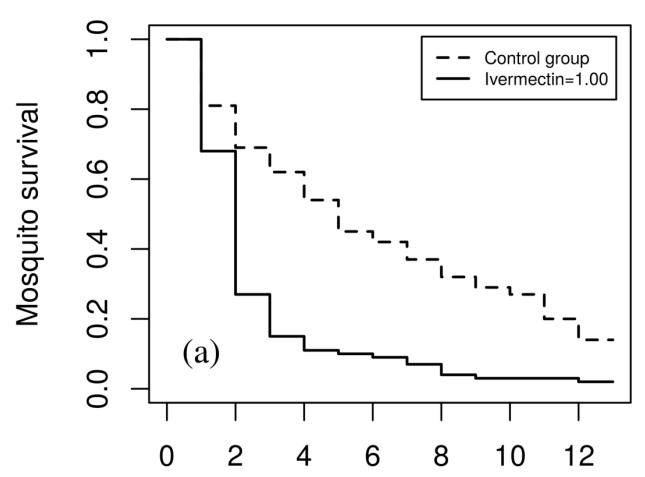

Day

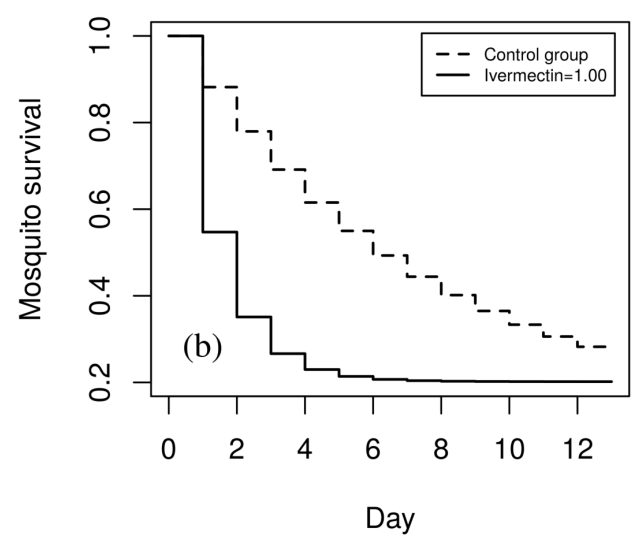

Day

Figure 6. Validation of the ivermectin model: (a) mosquito survival curve in empirical series as a result of ivermectin treatment in $100 \%$ of all human individuals when compared to the untreated human control group [22] and (b) mosquito survival curve as a result of model simulation of ivermectin treatment in $100 \%$ of all human individuals when compared to a model simulation with no treatment in the control group. 


\subsection{Towards Parameters for Assessing Malaria Incidence}

\subsubsection{Utility of Plasmodium Infection Metrics}

An important issue addressed in our model concerns the presentation of malaria burden results. For that purpose, we used a point analysis of annual malaria incidence per 100 inhabitants as a reliable metric of malaria transmission intensity. However, we also tested an alternate rate resulting from the combination of two other well-known and reliable infection rates: sporozoite rate $(Z)$ and parasite rate $(X)$.

We define the G-rate as the result from the product of both sporozoite and parasite rates. See Equation (6). With the background of different heterogeneity scenarios, we found a consistent correlation of the G-rate with malaria annual incidence, when compared to the established role of the parasite rate in defining the intensity of malaria transmission. Comparing the different plots in Figure 7, one sees that in the presence of transmission heterogeneity, malaria incidence correlates better with the G-rate, than with any of the other rates, $Z$ or $X$, separately.
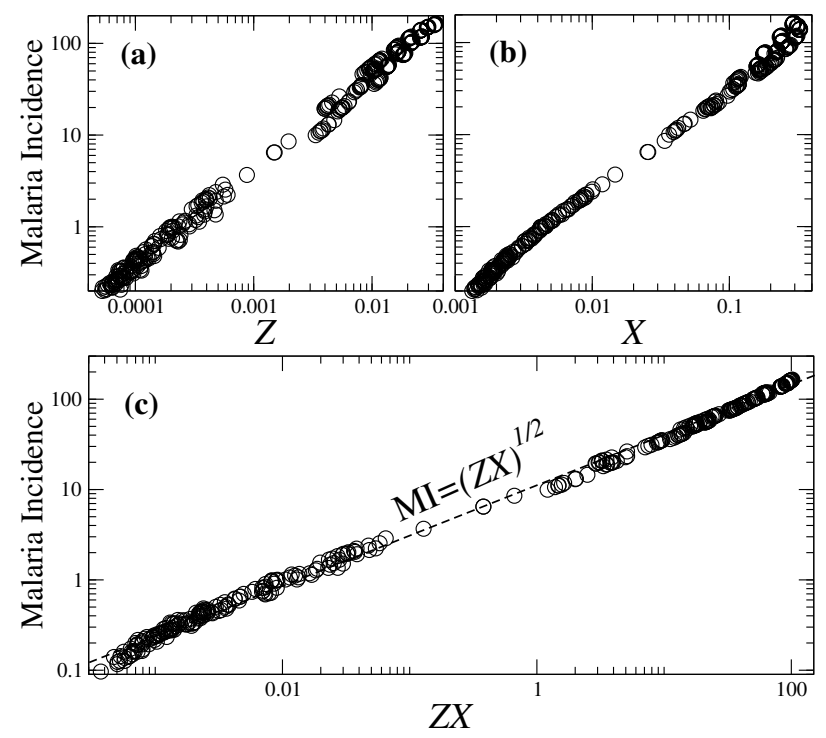

Figure 7. Scatter-plot between annual malaria incidence per 100 inhabitants and (a) the Z-rate, (b) the $X$-rate, and (c) the ZX-rate. In each case, we merge different levels of heterogeneity and at three different levels of ivermectin treatment, namely $p_{i v}=0.00,0.10$, and 0.20 . Independently of the heterogeneous affinity and ivermectin probability, there is a very accurate relationship given by $M I \sim \sqrt{Z X}$.

\subsection{Performance of Classical Ross-Macdonald Parameters in Heterogeneity-Driven Scenarios}

We determined the combined influence of ivermectin treatment on two important Ross-Macdonald model classical metrics, at different levels of heterogeneity affinity: the annual entomological inoculation rate (EIR), defined in Equation (7), and the basic reproductive number $\left(R_{0}\right)$, at different levels of heterogeneity (Figure 8a,b).

As expected, mosquito mortality progressively increases with higher percentual use of ivermectin in the general population. When the ivermectin treatment rate reaches the value of 0.20 , i.e., nearly $93 \%$ of all mosquito deaths occur during the 10 days after the initial blood meal, when the parasite is still developing into the next transmissible form, explaining the effectiveness of this drug in blocking disease transmission.

Nonetheless, our simulations indicate that EIR is a better indicator than $R_{0}$ in the prediction of malaria transmission, as shown in Figure 8c,d. A possible explanation for this observation is that EIR values are consistently higher with increasing levels of heterogeneity. 

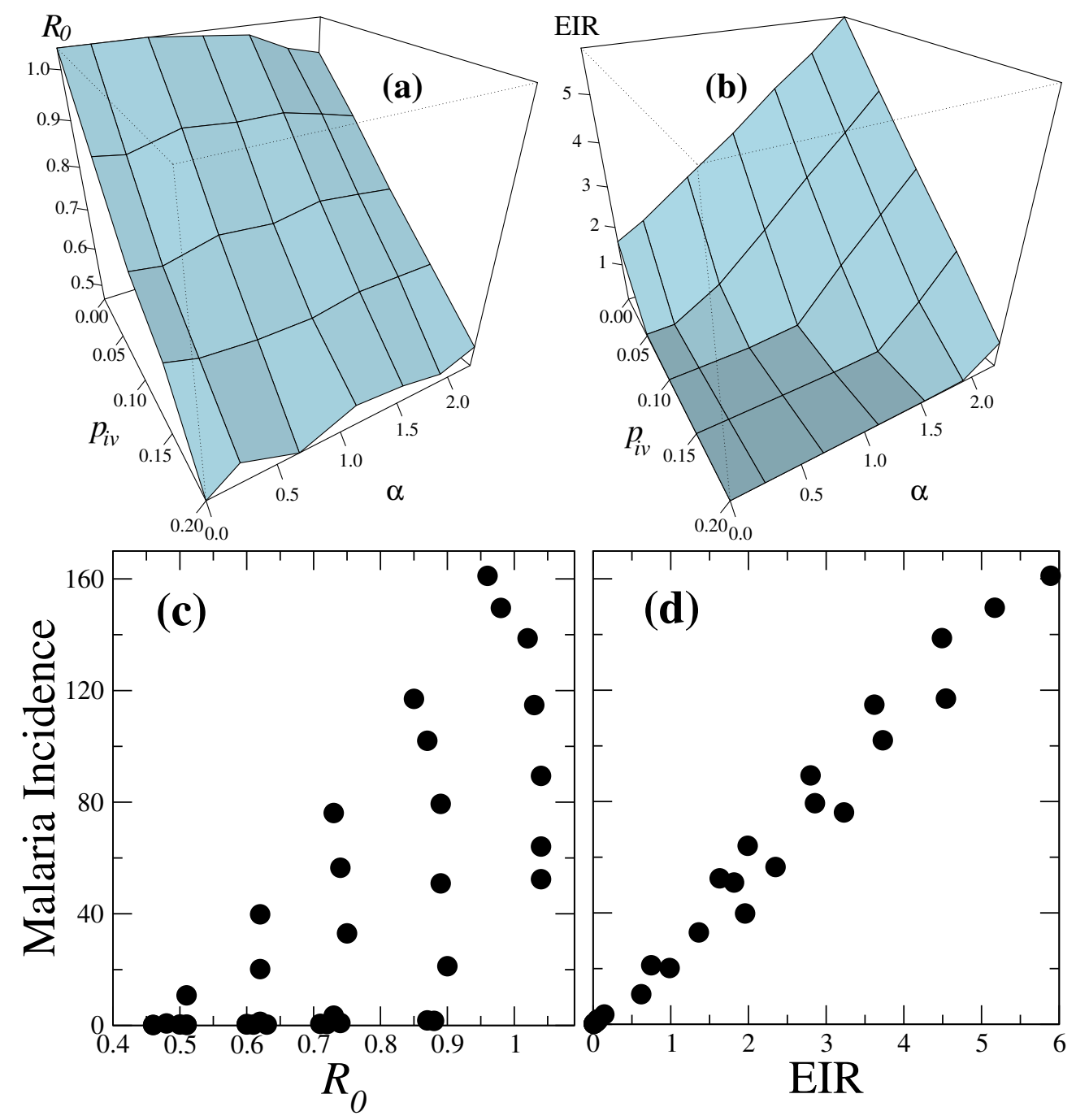

Figure 8. (a) The basic reproduction number, Equation (2), and (b) the annual entomological inoculation rate, Equation (7), both as a function of the ivermectin probability $p_{i v}$ and heterogeneous affinity $\alpha$. In (c,d), we show the scatter-plots of the basic reproduction number and annual entomological inoculation rate, respectively, with the corresponding values of malaria incidence. Clearly, there is a significantly stronger correlation of malaria incidence with $E I R$ than with $R_{0}$.

Additional simulations were performed for other classical Ross-Macdonald model parameters, such as the human feeding rate $a$, mosquito-to-human transmission parameter $b$, and human-to-mosquito transmission parameter $c$, with different results according to the heterogeneity level. While $a$ is not significantly affected by heterogeneity, we find a consistent reduction in $b$ with increasing levels of heterogeneity; on the contrary, $c$ is positively and linearly correlated with higher heterogeneity levels.

The level of heterogeneity at which $c$ became higher in magnitude than $b$ was above the level corresponding to the PW-rule of 20/60, where we had previously found an inversion of human infection prevalence. From this relation between $b$ and $c$, one may assume the possible existence of some form of proportionality of the fraction $\frac{c}{b}$ with the biting disparity index $\alpha$.

\section{Discussion and Conclusions}

We addressed heterogeneity as an important aspect in disease transmission, with a severe impact on the disease prevention strategy. To that end, we used a previously described agent-based malaria 
model to simulate malaria transmission in scenarios of varying heterogeneous affinity, aiming to identify parameters that potentiate transition from disease persistence to elimination. Emphasis was placed on the role of preventive interventions, such as mass administration of ivermectin treatment (possibly in combination with gametocidal therapy), as well as on the adaptability of field malaria transmission indicators to accurately predict malaria incidence.

Human-to-mosquito transmission, which is directly related to the time length of positive gametocytemia $\left(w_{h}\right)$, is fundamental in disease transmission. Mass administration of ivermectin can potentially inhibit transmission due to its lethal effect on mosquito populations, immediately after feeding, therefore not allowing for complete gametocyte maturation to take place inside the mosquito, long before completing the 10 day incubation time period. Such an effect is potentiated in the settings of high mosquito density and high human-to-mosquito transmission [22,24,35].

We found that in the presence of strong heterogeneity, the product between the sporozoite and the parasite infection rates becomes a reliable indicator for predicting malaria transmission, performing better than the parasite rate and sporozoite rate, separately, in identical heterogeneity settings, in particular when taking into consideration the possibility of mass administration of ivermectin. Furthermore, we found that among the classical Ross-Macdonald model metrics, EIR is much more sensitive than $R_{0}$ in predicting malaria incidence in high heterogeneity scenarios.

The assumptions of our model intend to assess the impact of heterogeneity in malaria transmission. Being an agent-based model, the assessment takes place at a qualitative level. In other words, while keeping its simplicity in order to facilitate the exploration of different scenarios, our results provide evidence, at least at a qualitative level, of the large efficiency of ivermectin administration. We described the quantitative output of our simulations, parameterized according to the knowledge about both populations and drug features, but carefully revised our claims, preventing stating quantitative conclusions, only possible with clinical trials, which is of course beyond the scope of a computational agent-based simulation.

Moreover, our model is based on a stochastic agent-based model that we previously published [24]. The model is implemented based in a detailed multi-parameter calibration, including information from empirical knowledge about mosquitoes, human-mosquito interaction, and the pharmacological features of ivermectin. Here, an additional assumption is derived from a well-established concept in economy and ecology, which is strongly connected to the presence of transmission hotspots and disease super-spreading events, as we now report in the manuscript: $20 \%$ of the causes are responsible for $80 \%$ of the consequences. Our model of heterogeneity was implemented as a mathematical tool replicating that concept, in accordance to the use of the 20/80 rule in infectious disease research by Woolhouse, in 1996.

To better explore the robustness of our results against variations of heterogeneous affinity, we introduced a parameter that measures theat affinity. Our results show a linear relationship between the level of heterogeneous affinity and the minimal level of ivermectin administration. Again, the specific linear coefficients are not taken as trustworthy results, but the linear relationship between the minimum level of drug administration and the level of heterogeneity can be presented as one conclusion in this study.

One particular aspect uncovered in our simulations is the evidence that the annual entomological inoculation rate seems to be a better predictor than standard quantities such as the basic reproduction number.

Overall, our model emphasizes the importance of taking into account heterogeneity, when predicting malaria transmission, showing that the effect of mass drug administration of ivermectin is not only dependent on ivermectin intensity and human-to-mosquito transmission efficiency, but that it is also clearly influenced by the presence of heterogeneity in disease transmission.

Heterogeneity is clearly illustrated by the presence of transmission hotspots, which have been shown to be geographically distinct and able to present substantial diversity in temporal transmission dynamics [47]. The role of these hotspots in malaria transmission may assume special importance 
in small villages in close proximity to high Anopheles mosquito density and active larval breeding sites. Children, as well as recent immigrants coming from malaria high-risk regions should also be considered as transmission hotspot heterogeneity sources. In our view, a combined treatment strategy with gametocidal drugs (such as artemisinin, primaquine, or methylene blue) and mass administration of ivermectin, specifically targeting transmission hotspots, may stand as a promising alternative to dealing with the risk of malaria resurgence in high heterogeneity settings, deserving further consideration in future field trials.

Author Contributions: J.S. implemented the model and performed the simulations. J.S. and P.G.L. structured the text and produced the figures. J.L. and A.M.M. assisted with background ideas, and the overall research was coordinated by J.S. and P.G.L. All authors revised and contributed to the final version of the manuscript.

Funding: This research received no external funding.

Acknowledgments: We thank Miguel Prudêncio (IMM-Lisbon) for his help in defining model parameters and for his crucial remarks in discussing the model design.

Conflicts of Interest: No competing interests apply to this manuscript.

\section{References}

1. UNICEF/WHO. Reversing the Incidence of Malaria 2000-2015. In WHO Global Malaria Programme; World Health Organization: Geneva, Switzerland, 2015; pp. 1-40

2. World Health Organization. World Malaria Report; World Health Organization: Geneva, Switzerland, 2019.

3. Acevedo, M.A.; Prosper, O.; Lopiano, K.; Ruktanonchai, N.; Caughlin, T.T.; Martcheva, M.; Osenberg, C.W.; Smith, D.L. Spatial heterogeneity, host movement and mosquito-borne disease transmission. PLoS ONE 2015, 10, e0127552. [CrossRef] [PubMed]

4. Malede, A.; Alemu, K.; Aemero, M.; Robele, S.; Kloos, H. Travel to farms in the lowlands and inadequate malaria information significantly predict malaria in villages around Lake Tana, northwest Ethiopia: A matched case-control study. Malar. J. 2018, 17, 290. [CrossRef] [PubMed]

5. Mukabana, W.R.; Takken, W.; Killeen, G.F.; Knols, B.G.J. Allomonal effect of breath contributes to differential attractiveness of humans to the African malaria vector Anopheles gambiae. Malar. J. 2004, 3, 1. [CrossRef] [PubMed]

6. Smith, D.L.; McKenzie, F.E.; Snow, R.W.; Hay, S.I. Revisiting the basic reproductive number for malaria and its implications for malaria control. PLoS Biol. 2007, 5, e42. [CrossRef] [PubMed]

7. Van der Waal, K.L.; Ezenwa, V.O. Heterogeneity in pathogen transmission: Mechanisms and methodology. Funct. Ecol. 2016, 30, 1606-1622. [CrossRef]

8. Cooper, L.; Kang, S.Y.; Bisanzio, D.; Maxwell, K.; Rodriguez-Barraquer, I.; Greenhouse, B.; Drakeley, C.; Arinaitwe, E.; Staedke, S.G.; Gething, P.W.; et al. Pareto rules for malaria super-spreaders and super-spreading. Nat. Commun. 2019, 10, 1-9.

9. Obala, A.A.; Kutima, H.L.; Nyamogoba, H.D.N.; Mwangi, A.W.; Simiyu, C.J.; Magak, G.N.; Khwa-Otsyula, B.O.; Ouma, J.H. Anopheles gambiae and Anopheles arabiensis population densities and infectivity in Kopere village, Western Kenya. J. Infect. Dev. Ctries. 2012, 6, 637-643. [CrossRef]

10. Dekker, T.; Takken, W.; Knols, B.G.J.; Bouman, E.; van de Laak, S.; de Bever, A.; Huisman, P.W.T. Selection of biting sites on a human host by Anopheles gambiae s. s., An. arabiensis and An. quadriannulatus. Entomol. Exp. Appl. 1998, 87, 295-300. [CrossRef]

11. Mahande, A.; Mosha, F.; Mahande, J.; Kweka, E. Feeding and resting behavior of malaria vector, Anopheles arabiensis with reference to zooprophylaxis. Malar. J. 2007, 6, 100. [CrossRef]

12. Akogbéto, M.C.; Salako, A.S.; Dagnon, F.; Aikpon, R.; Kouletio, M.; Sovi, A.; Sezonlin, M. Blood feeding behavior comparison and contribution of Anopheles coluzzii and Anopheles gambiae, two sibling species living in sympatry, to malaria transmission in Alibori and Donga region, northern Benin, West Africa. Malar. J. 2018, 17, 307. [CrossRef]

13. Cornet, S.; Nicot, A.; Rivero, A.; Gandon, S. Both infected and uninfected mosquitoes are attracted toward malaria infected birds. Malar. J. 2013, 12, 179. [CrossRef] 
14. De Moraes, C.M.; Stanczyk, N.M.; Betz, H.S.; Pulido, H.; Sim, D.G.; Read, A.F.; Mescher, M.C. Malaria-induced changes in host odors enhance mosquito attraction. Proc. Natl. Acad. Sci. USA 2014, 111, 11079-11084. [CrossRef]

15. Lacroix, R.; Mukabana, W.R.; Gouagna, L.C.; Koella, J.C. Malaria infection increases attractiveness of humans to mosquitoes. PLoS Biol. 2005, 3, 1590-1593. [CrossRef]

16. Woolhouse, M.E.; Dye, C.; Etard, J.F.; Smith, T.; Charlwood, J.D.; Garnett, G.P.; Hagan, P.; Hii, J.L.K.; Ndhlovu, P.D.; Quinnell, R.J.; et al. Heterogeneities in the transmission of infectious agents: Implications for the design of control programs. Proc. Natl. Acad. Sci. USA 1997, 94, 338-342. [CrossRef]

17. Pareto, V. Manuale di Economia Politica con una Introduzione Alla Scienza Sociale; Società Editrice Libraria: Torino, Italy, 1919; Volume 13.

18. Brady, O.J.; Slater, H.C.; Pemberton-Ross, P.; Wenger, E.; Maude, R.J.; Ghani, A.C.; Penny, M.A.; Gerardin, J.; White, L.J.; Chitnis, M.; et al. Role of mass drug administration in elimination of Plasmodium falciparum malaria: A consensus modelling study. Lancet Glob. Health 2017, 5, e680-e687. [CrossRef]

19. Bousema, T.; Drakeley, C.; Gesase, S.; Hashim, R.; Magesa, S.; Mosha, F.; Otieno, S.; Carneiro, I.; Cox, J.; Msuya, E.; et al. Identification of hot spots of malaria transmission for targeted malaria control. J. Infect. Dis. 2010, 201, 1764-1774. [CrossRef]

20. Bousema, T.; Griffin, J.T.; Sauerwein, R.W.; Smith, D.L.; Churcher, T.S.; Takken, W.; Ghani, A.; Drakeley, C.; Gosling, R. Hitting hotspots: Spatial targeting of malaria for control and elimination. PLoS Med. 2012, 9, e1001165. [CrossRef]

21. Noé, A.; Zaman, S.I.; Rahman, M.; Saha, A.K.; Aktaruzzaman, M.M.; Maude, R.J. Mapping the stability of malaria hotspots in Bangladesh from 2013 to 2016. Malar. J. 2018, 17, 259. [CrossRef]

22. Chaccour, C.; Lines, J.; Whitty, C.J.M. Effect of Ivermectin on Anopheles gambiae Mosquitoes Fed on Humans: The Potential of Oral Insecticides in Malaria Control. J. Infect. Dis. 2010, 202, 113-116. [CrossRef]

23. Slater, H.C.; Foy, B.D.; Kobylinski, K.; Chaccour, C.; Watson, O.J.; Hellewell, J.; Aljayyoussi, G.; Bousema, T.; Burrows, J.; D'Alessandro, U. Ivermectin as a novel complementary malaria control tool to reduce incidence and prevalence: A modelling study. Lancet Infect. Dis. 2020, 20, P498-P508. [CrossRef]

24. Sequeira, J.; Louçã, J.;Mendes, A.M.; Lind, P.G. Transition from endemic behavior to eradication of malaria due to combined drug therapies: An agent-model approach. J. Theor. Biol. 2020, 484, 110030. [CrossRef]

25. Macdonald, G.; The analysis of equilibrium in malaria. Trop. Dis. Bull. 1952, 49, 813-829.

26. Ross, R.; Some a Priori Pathometric Equations. Br. Med. J. 1915, 1, 546-547. [CrossRef]

27. Biggs, J.; Raman, J.; Cook, J.; Hlongwana, K.; Drakeley, C.; Morris, N.; Serocharan, I.; Agubuzo, E.; Kruger, P.; Mabuza, A.; et al. Serology reveals heterogeneity of Plasmodium falciparum transmission in northeastern South Africa: Implications for malaria elimination. Malar. J. 2017, 16, 48. [CrossRef]

28. Dye, C.; Hasibeder, G. Population dynamics of mosquito-borne disease: Effects of flies which bite some people more frequently than others. Trans. R. Soc. Trop. Med. Hyg. 1986, 80, 69-77. [CrossRef]

29. Alemu, K.; Worku, A.; Berhane, Y.; Kumie, A. Spatiotemporal clusters of malaria cases at village level, northwest Ethiopia. Malar. J. 2014, 13, 1-8. [CrossRef]

30. Camarda, G.; Jirawatcharadech, P.; Priestley, R.S.; Saif, A.; March, S.; Wong, M.H.; Leung, S.; Miller, A.B.; Baker, D.A.; Alano, P.; et al. Antimalarial activity of primaquine operates via a two-step biochemical relay. Nat. Commun. 2019, 10, 3226. [CrossRef]

31. Lawpoolsri, S.; Klein, E.Y.; Singhasivanon, P.; Yimsamran, S.; Thanyavanich, N.; Maneeboonyang, W.; Hungerford, L.L.; Maguire, J.H.; Smith, D.L. Optimally timing primaquine treatment to reduce Plasmodium falciparum transmission in low endemicity Thai-Myanmar border populations. Malar. J. 2009, 8, 159. [CrossRef]

32. Shekalaghe, S.; Mosha, D.; Hamad, A.; Mbaga, T.A.; Mihayo, M.; Bousema, T.; Drakeley, C.; Abdulla, S. Optimal timing of primaquine to reduce Plasmodium falciparum gametocyte carriage when co-administered with artemether-lumefantrine. Malar. J. 2020, 19, 34. [CrossRef]

33. Bosson-Vanga, H.; Franetich, J.F.; Soulard, V.; Sossau, D.; Tefit, M.; Kane, B.; Vaillant, J.C.; Borrmann, S.; Müller, O.; Dereuddre-Bosquet, N.; et al. Differential activity of methylene blue against erythrocytic and hepatic stages of Plasmodium. Malar. J. 2018, 17, 143. [CrossRef]

34. Mendes, A.M.; Albuquerque, I.S.; Machado, M.; Pissarra, J.; Meireles, P.; Prudêncio, M. Inhibition of Plasmodium liver infection by ivermectin. Antimicrob. Agents Chemother. 2017, 61, 1-8. [CrossRef] [PubMed] 
35. Kobylinski, K.C.; Foy, B.D.; Richardson, J.H. Ivermectin inhibits the sporogony of Plasmodium falciparum in Anopheles gambiae. Malar. J. 2012, 11, 381. [CrossRef] [PubMed]

36. Chaccour, C.; Rabinovich, N.R. Advancing the repurposing of ivermectin for malaria. Lancet 2019, 393, 1480-1481. [CrossRef]

37. Smit, M.R.; Ochomo, E.O.; Aljayyoussi, G.; Kwambai, T.K.; Abong'o, B.O.; Chen, T.; Bousema, T.; Slater, H.C.; Waterhouse, D.; Bayoh, N.M. Safety and mosquitocidal efficacy of high-dose ivermectin when co-administered with dihydroartemisinin-piperaquine in Kenyan adults with uncomplicated malaria (IVERMAL): A randomised, double-blind, placebo-controlled trial. Lancet Infect. Dis. 2018, 18, P615-P626. [CrossRef]

38. Foy, B.D.; Alout, H.; Seaman, J.A.; Rao, S.; Magalhaes, T.; Wade, M.; Parikh, S.; Soma, D.D.; Sagna, A.D.; Fournet, F. Efficacy and risk of harms of repeat ivermectin mass drug administrations for control of malaria (RIMDAMAL): A cluster-randomised trial. Lancet 2019, 393, P1517-P1526. [CrossRef]

39. Ouédraogo, A.L.; Bastiaens, G.J.; Tiono, A.B.; Guelbéogo, W.M.; Kobylinski, K.C.; Ouédraogo, A.; Barry, A.; Bougouma, E.C.; Nebie, I.; Ouattara, M.S.; et al. Efficacy and safety of the mosquitocidal drug ivermectin to prevent malaria transmission after treatment: A double-blind, randomized, clinical trial. Clin. Infect. Dis. 2015, 60, 357-365. [CrossRef]

40. Azevedo, R.; Mendes, A.M.; Prudêncio, M. Inhibition of Plasmodium sporogonic stages by ivermectin and other avermectins. Parasites Vectors 2019, 12, 549. [CrossRef]

41. Stresman, G.H.; Giorgi, E.; Baidjoe, A.; Knight, P.; Odongo, W.; Owaga, C.; Shagari, S.; Makori, E.; Stevenson, J.; Drakeley, C.; et al. Impact of metric and sample size on determining malaria hotspot boundaries. Sci. Rep. 2017, 7, 45849. [CrossRef]

42. Smith, D.L.; McKenzie, F.E. Statics and dynamics of malaria infection in Anopheles mosquitoes. Malar. J. 2004, 3, 13. [CrossRef]

43. Jensen, T.P.; Bukirwa, H.; Njama-Meya, D.; Francis, D.; Kamya, M.R.; Rosenthal, P.J.; Dorsey, G. Use of the slide positivity rate to estimate changes in malaria incidence in a cohort of Ugandan children. Malar. J. 2009, 8, 213. [CrossRef]

44. Boyce, R.M.; Reyes, R.; Matte, M.; Ntaro, M.; Mulogo, E.; Lin, F.C.; Siedner, M.J. Practical implications of the non-linear relationship between the test positivity rate and malaria incidence. PLOS ONE 2016, 11, e0152410. [CrossRef] [PubMed]

45. Bi, Y.; Hu, W.; Liu, H.; Xiao, Y.; Guo, Y.; Chen, S.; Zhao, L.; Tong, S. Can slide positivity rates predict malaria transmission? Malar. J. 2012, 11, 117. [CrossRef] [PubMed]

46. Pombi, M.; Calzetta, M.; Guelbeogo, W.M.; Manica, M.; Perugini, E.; Pichler, V.; Mancini, E.; Sagnon, N.F.; Ranson, H.; Della Torre, A. Unexpectedly high Plasmodium sporozoite rate associated with low human blood index in Anopheles coluzzii from a LLIN-protected village in Burkina Faso. Sci. Rep. 2018, 8, 12806. [CrossRef] [PubMed]

47. Bejon, P.; Williams, T.N.; Liljander, A.; Noor, A.M.; Wambua, J.; Ogada, E.; Olotu, A.; Osier, F.H.; Hay, S.I.; Färnert, A. Stable and unstable malaria hotspots in longitudinal cohort studies in Kenya. PLoS Med. 2010, 7, e1000304. [CrossRef] [PubMed]

Publisher's Note: MDPI stays neutral with regard to jurisdictional claims in published maps and institutional affiliations.

(C) 2020 by the authors. Licensee MDPI, Basel, Switzerland. This article is an open access article distributed under the terms and conditions of the Creative Commons Attribution (CC BY) license (http:// creativecommons.org/licenses/by/4.0/). 